\title{
Tracheal transection after blunt trauma by motor vehicle accident: A diagnostic and survival challenge
}

\author{
A.K. Qumrul Huda ${ }^{1}$, Rezwanul Hoque ${ }^{2}$, Wong Poo Sing ${ }^{3}$
}

\begin{abstract}
Tracheal transection is a rare injury after blunt trauma. Complete disruption of the trachea is rare, with a reported incidence of 1 in 4491 trauma admission. Blind oral or nasal intubation in patients with tracheal injury can lead to false extra passage. The presence of tracheal transection in the intubated ventilating patient is rarer and constitutes a major diagnostic and survival challenge. Here we present a case of tracheal transection caused by motor vehicle accident diagnosed few days later of the incidence by Computed tomography (CT) of chest, who went for thoracotomy and anastomosis of tracheal transection but developed anastomosis dehiscence. Repeated trial for anastomosis could not save the life of the patient because of development of severe sepsis, disseminated intravascular coagulation (DIC) and multi organ dysfunction syndrome (MODS). Early diagnosis by CT of chest and tracheal anastomotic surgery as early as possible (possibly within $24 \mathrm{hrs}$ ) before development of sepsis from mediastinitis is paramount for survival of patient.
\end{abstract}

Key words: Tracheal transection, Computed tomography (CT) of chest, Thoracotomy, Sepsis.

\section{Introduction}

Tracheal transection is a rare injury after blunt trauma. Complete disruption of the trachea is rare, with a reported incidence of 1 in 4491 trauma admission. ${ }^{1}$ Blind oral or nasal intubation in patients with tracheal injury can lead to false extra tracheal intubation, with irretrievable loss of the airway. The presence of tracheal transection in the intubated, ventilating patient is rarer and constitutes a major diagnostic and survival challenge. The liberal use of computed tomography (CT) Scan as an adjunct to endoscopy is paramount.

\section{Case report}

The unfortunate 50 year old medical doctor met with a motor vehicle accident in Dhaka, Bangladesh. He sustained bilateral rib fractures and lung contusion, fracture of left mid humerus and left femur with a deep open wound in the left calf. Right chest drain followed by left were inserted. His breathing was labored and required immediate intubation and was put on ventilator. He became hyperpyrexic $\left(40^{\circ} \mathrm{C}\right)$ and urine output gradually diminished with rising serum creatinine. There was extensive surgical emphysema from head to groin. Samples for culture were taken from blood, urine and endotracheal tube (ET) secretion and he was commenced on i.v Tazobactum +

1 Dr. A K Qumrul Huda, MD (Anesthesiology), Associate Professor (ICU), Department of Anesthesia, Analgesia and Intensive Care Medicine, BSMMU, Dhaka

2 Dr. Rezwanul Hoque, FCPS (Surgery), MS (Cardiothoracic Surgery), FRCS (Edinburgh, Glasgow), Associate Professor, Department of Cardiothoracic Surgery, BSMMU, Dhaka

3 Dr. Wong Poo Sing, FRCS (Edin), FRCS (Cardiothoracic Surgery, Edin), FCCP, FAMS, Mount Elizabeth Medical Centre, Singapore

\section{Corresponding Author:}

Dr. A K Qumrul Huda

Associate Professor (ICU), Dept of Anesthesiology

Bangabandhu Sheikh Mujib Medical University (BSMMU)

Shahbag, Dhaka-1000, Bangladesh

Email: qhuda309@gmail.com piperacillin $4.5 \mathrm{gm}$ tds. CT scan of chest showed multiple bilateral rib fractures which was delineated by plain chest Xray earlier. There was a near complete transection of the distal trachea $2.5 \mathrm{~cm}$ proximal to the carina with extensive mediastinal, chest wall and neck surgical emphysema.
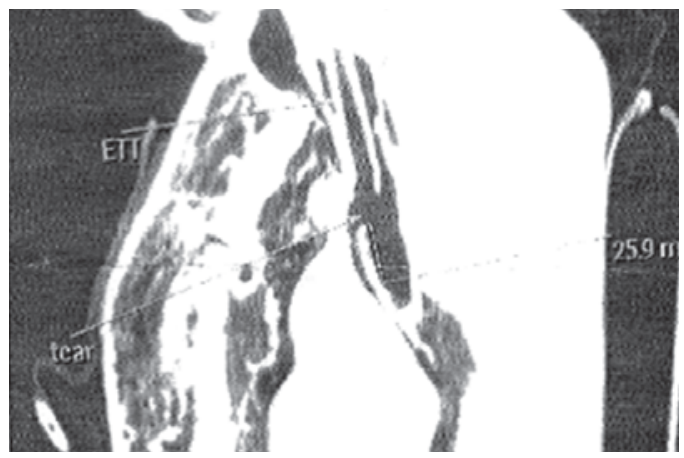

Figure 1: CT image showing longitudinal section of trachea transected at the level $1 \mathrm{~cm}$ above carina

The fractures were managed by internal fixation and external stabilization as required. He was given platelet transfusion, fresh frozen plasma and other resuscitation supportive treatment. The patient was transferred to operation theatre.

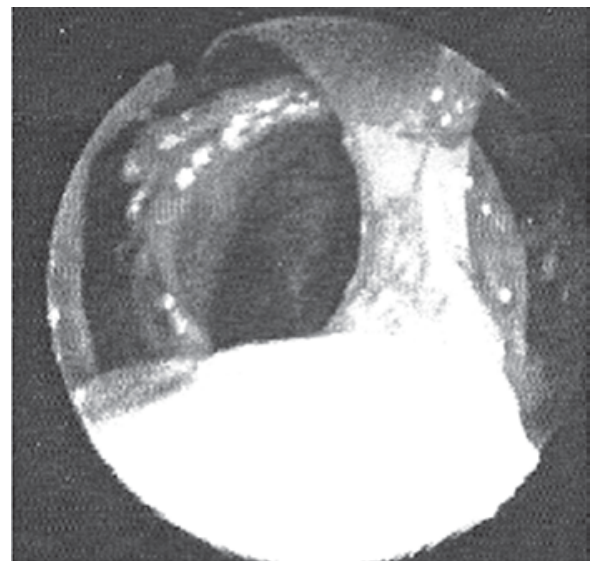

Figure 2: Bronchoscopic view of tracheal transection before surgery 
Flexible Bronchoscopy showed near total transection of the distal trachea just beyond the tip of the ET tube. A right thoracotomy was performed and tracheal transection was identified. There was pus and sent for bacteriology examination.

The area was debrided and edges of the trachea were fashioned and anastomosis was performed. The repair was tested with $25 \mathrm{~cm}$ of water pressure and was found to be air tight.

His neck was kept in a flexed position to prevent tension on the tracheal suture line.

After operation (24hrs Appx.), the patient's sepsis deteriorated to septic shock and that was evidenced by low urine output with high creatinine and multiple gram negative (mostly) organisms (Klebsiella, Acinetobacter, Proteus, Pseudomonas, Streptococcus, Enterococcus ) were isolated from mediastinal swab, blood, tracheal aspirate and urinary catheter tip. The antibiotic Tigecycline and polymixin-B were sensitive and given. But the septicemia was not resolved rather growing worse which was evidenced by persistent fever $\left(>41^{\circ} \mathrm{c}\right)$, thick purulent huge secretion from tracheostomy tube. Air leakage through anterior chest drain suspected for tracheal anastomosis defects and confirmed by flexible bronchoscopy and attempts taken for repair once again. But it failed to maintain oxygenation. Finally through cardio pulmonary bypass the repair was made at 10 and 2 o' clock position of the trachea. But in spite of all efforts patient went to irreversible shock and succumbed to severe sepsis and it's inevitable consequences.

\section{Discussion:}

Complete disruption of the cervical trachea is rare, with a reported incidence of 1 in 4491 trauma admission. ${ }^{1}$ About $80 \%$ of the tracheal injuries occur within $2.5 \mathrm{~cm}$ of the carina. ${ }^{2}$ Men are more affected than women in a ratio of five to one with a mean age of late 20 s to mid 30 s. A high index of suspicion exist in any injury that may be associated with airway trauma. Signs and symptoms may be subtle in tracheal injuries and also relatively non- specific co-relating poorly with the severity of the underlying injuries. ${ }^{3}$ Blind oral or nasal intubation in patients with tracheal injury can lead to false extra tracheal intubation, with irretrievable loss of airway. In one large series of patients with blunt injuries to cervical trachea, blind intubation failed to achieve an adequate airway in $76 \%$. Intubation over a fibreoptic bronchoscope has been advocated as an aid to diagnosis of upper airway injury and act as a stent to guide the endotracheal tube in to distal trachea. ${ }^{4}$

Though mediastinal emphysema is commonly seen on the $\mathrm{X}$-ray, ${ }^{5}$ particular diagnostic difficulties arise in the trauma patient with out on going post intubation ventilatory problem. Initially diagnosis of lower tracheal transection was missed in this case but suspicion arose by severe subcutaneous emphysema from head to groin, ${ }^{6}$ with progression of high rise of temperature $\left(41^{\circ} \mathrm{C}\right)$ by the $5^{\text {th }}$ day of incidence, which was obvious finding of severe sepsis from mediastinitis. Due to delayed diagnosis of tracheal transection, chance of survival is seriously compromised and death may be due to severe sepsis, DIC and MODS. In one study it is seen that after repair of tracheal transection, there is a $14 \%$ to $25 \%$ mortality mainly because of delayed diagnosis and consequently worse outcome. ${ }^{7}$ Connecting the endotracheal tube to positive pressure ventilation can produce major air leakage causing severe mediastinal emphysema, which should be arisen some suspicion about the tracheal transection and should go for CT of chest without any delay.

After complete transection of the trachea, an intact sheath of tracheal fascia may allow a conscious patient to breath. ${ }^{6,8,9} \mathrm{In}$ this case the tip of the endotracheal tube was above the tracheal transection but the tracheal continuity of the injured trachea was maintained by small bridge of intact tissue. This demonstrates that adequate ventilation and even apparently correct position of the endotracheal tube are no guarantee of the integrity of the upper airway. If CT Scan of chest had been advised earlier before development of sepsis from mediastinitis, the diagnosis of tracheal transection would have been made earlier and so the tracheal anastomotic surgery also. In that case there had been more chances of survival without developing mediastinitis. We strongly advise CT scan of chest as a reliable diagnostic modality in suspected severe tracheal injury, particularly in the intubated patient. We also feel that the presence of massive subcutaneous emphysema in the absence of significant pneumothoraces and minimal air leak from the intercostal water seal drainage should have alerted us to the possibility of tracheal injury.

\section{Acknowledgement}

Thanks to Department of Cardiothoracic Surgery and Critical Care Unit of Gleneagle Hospital, Singapore for management of the patient.

\section{References}

1. Ford HR, Gardner MJ, Lynch JM. Laryngo tracheal disruption from blunt pediatric neck injuries: impact of early recognition and intervention on outcome. J of paediatrsurg 1995; 30: 331-4.

2. Coventry BJ, Peacock MJ. Survival after traumatic complete transection of the trachea. Aust N Z J Surg1997; 67: 388-90.

3. Rossback MM, Johnson SB, Gomez MA, et al. Management of major tracheobronchial injuries. A 28 year experience. Ann Thorac surg 1998; 65: 182-6.

4. Baumgartner FJ, Ayres B, Theurer C . Dangers of false intubation after traumatic tracheal transection. Ann Thorac Surg 1997; 63: 227-8.

5. Andrew D Berston, Neil Soni. Intensive care manual edn $5^{\text {th }} 2003$; 67: chest injuries, GM Clark: 723.

6. Hosny A, Bhendwal S, Hosni A, Transection of cervical trachea following blunt trauma. J Laryngol Otol 1995; 109: 250-1.

7. Robert B, Lee MD. Traumatic injury of the Cervicothoracic trachea and major bronchi. Chest Surg Clin North Am 1997; 72: 285-304.

8. Hermon A, Segal K, Har-El G, Abraham A, Sidi j. Complete cricotracheal separation following blunt trauma to the neck. J Trauma 1987; 27: 1365-7.

9. Baumgartner F, Sheppard B, de Virgilio C, Esrig B, Harrier D, Nelson RJ et al. Tracheal and main bronchial disruptions after blunt chest trauma: presentation and management. Ann Thorac Surg 1990; 50: 569-74 